\title{
Approximate Series Solution of Nonlinear Singular Boundary Value Problems Arising in Physiology
}

\author{
Randhir Singh, Jitendra Kumar, and Gnaneshwar Nelakanti \\ Department of Mathematics, Indian Institute of Technology Kharagpur, Kharagpur 721302, India \\ Correspondence should be addressed to Randhir Singh; randhir.math@gmail.com
}

Received 8 August 2013; Accepted 9 January 2014; Published 20 February 2014

Academic Editors: P. Bi, H. Su, and S. A. Tersian

Copyright (c) 2014 Randhir Singh et al. This is an open access article distributed under the Creative Commons Attribution License, which permits unrestricted use, distribution, and reproduction in any medium, provided the original work is properly cited.

\begin{abstract}
We introduce an efficient recursive scheme based on Adomian decomposition method (ADM) for solving nonlinear singular boundary value problems. This approach is based on a modification of the ADM; here we use all the boundary conditions to derive an integral equation before establishing the recursive scheme for the solution components. In fact, we develop the recursive scheme without any undetermined coefficients while computing the solution components. Unlike the classical ADM, the proposed method avoids solving a sequence of nonlinear algebraic or transcendental equations for the undetermined coefficients. The approximate solution is obtained in the form of series with easily calculable components. The uniqueness of the solution is discussed. The convergence and error analysis of the proposed method are also established. The accuracy and reliability of the proposed method are examined by four numerical examples.
\end{abstract}

\section{Introduction}

In this paper we propose an efficient recursive scheme based on Adomian decomposition method (ADM) for solving a class of nonlinear singular boundary value problems (SBVPs) that arising in physiology [1-5]:

$$
\begin{gathered}
\left(x^{\alpha} y^{\prime}(x)\right)^{\prime}=x^{\alpha} f(x, y(x)), \quad 0<x<1, \alpha \geq 0, \\
y^{\prime}(0)=0, \quad \text { ay }(1)+b y^{\prime}(1)=c .
\end{gathered}
$$

Here $a>0, b \geq 0$, and $c$ are any finite real constants. We assume that for $(x, y) \in\{(0,1) \times \mathbb{R}\}$, the functions $f(x, y)$ and $\partial f / \partial y$ are continuous and $\partial f / \partial y \geq 0$. Singular boundary value problems frequently arise in the modeling of many problems in biological, physical, and engineering sciences. For example, it arises in the study of steady-state oxygen diffusion in a spherical cell with Michaelis-Menten uptake kinetics [6] with $\alpha=2$ and $f(x, y)=N_{1} y / y+K_{1}$, where $N_{1}$ and $K_{1}$ are positive real constants. In heat conduction model in human head $[7,8]$ with $\alpha=2, f(x, y)=-a_{0} e^{-b_{0} y}$, where $a_{0}$ and $b_{0}$ are positive reals.

There is considerable literature on the numerical treatment of singular boundary value problems [1-11] and many of the references therein. The main difficulty of the problem (1) is that the singularity behavior occurs at $x=0$. Various efficient numerical techniques have been used to deal with such SBVPs, such as finite difference method (FDM) [9], cubic spline method (CSM) [1], and B-spline method (BSM) [2]. Although, these techniques are efficient and have many advantages, a huge amount of computational work is needed which combines some root-finding methods to obtain accurate numerical solution especially for nonlinear SBVPs. Recently, some newly developed approximate methods were also applied to deal with such SBVPs, such as variational iteration method (VIM) [5, 11], Adomian decomposition method (ADM) [3], and modified Adomian decomposition method (MADM) [4]. It is well known that solving nonlinear two-point boundary value problems (BVPs) using $\mathrm{ADM} / \mathrm{MADM}$ is always a computationally involved task. Since it requires computation of unknown constants in a sequence of nonlinear or more difficult transcendental equations. Moreover, in some cases these unknown constants may not be uniquely determined and this may be the major disadvantage of ADM/MADM for solving nonlinear BVPs.

The aim of this work is to introduce an efficient recursive scheme to overcome the difficulties that occur in the ADM or MADM for solving nonlinear SBVPs (1). This approach 
is based on a modification of the ADM; here we use all the boundary conditions to derive an integral equation before establishing the recursive scheme for the solution components. In fact, we develop the recursive scheme without any undetermined coefficients while computing the solution components. Unlike the ADM, the proposed scheme avoids solving a sequence of nonlinear algebraic or transcendental equations for the undetermined coefficients. The approximations of the solution are obtained in the form of series with easily computable components. The sufficient condition that guarantees the existence of a unique solution of the problem (1) is proved. The convergence analysis and the error estimation are also discussed. Finally, the accuracy and reliability of the proposed method are examined by four numerical examples.

1.1. The Review of $A D M$. In this subsection, we briefly describe the ADM for solving SBVPs (1). Recently, many researchers [3, 4, 12-23] have applied the ADM to deal with many different scientific models. According to the ADM we rewrite (1) in a operator form as

$$
L y(x)=f(x, y(x)),
$$

where $L=x^{-\alpha} d / d x\left(x^{\alpha} d / d x\right)$ is a second-order linear differential operator. In [24] Wazwaz defines the inverse operator $L^{-1}$ as

$$
L^{-1}[\cdot]=\int_{0}^{x} x^{-\alpha} \int_{0}^{x} x^{\alpha}[\cdot] d x d x
$$

Operating $L^{-1}[\cdot]$ on both sides of (2) and using the condition $y^{\prime}(0)=0$, we have

$$
y(x)=y(0)+L^{-1}[f(x, y(x))] .
$$

The main idea of the ADM depends on decomposing the solution $y(x)$ and the nonlinear function $f(x, y(x))$ by an infinite series as

$$
y(x)=\sum_{j=0}^{\infty} y_{j}(x), \quad f(x, y(x))=\sum_{j=0}^{\infty} A_{j},
$$

where $A_{j}$ are Adomian's polynomials which can be constructed for various classes of nonlinear functions with the formula given in [13] as

$$
A_{n}=\frac{1}{n !} \frac{d^{n}}{d \lambda^{n}}\left[f\left(x, \sum_{k=0}^{\infty} y_{k} \lambda^{k}\right)\right]_{\lambda=0}, \quad n=0,1,2, \ldots
$$

Substituting the series (5) into (4), we obtain

$$
\sum_{j=0}^{\infty} y_{j}(x)=y(0)+L^{-1}\left[\sum_{j=0}^{\infty} A_{j}\right] \text {. }
$$

Upon matching both sides of (7), the ADM is given by

$$
y_{0}(x, \eta)=\eta, \quad y_{j}(x, \eta)=L^{-1}\left[A_{j-1}\right], \quad j \geq 1,
$$

where $y(0)=\eta \neq 0$ is unknown constant to be determined. Having determined the components $y_{n}(x, \eta)$, the series solution of $y(x)$ follows immediately with the undetermined $\eta$. Hence, the $n$-term truncated series solution is given as

$$
\phi_{n}(x, \eta)=\sum_{j=0}^{n} y_{j}(x, \eta)
$$

It should be noted that the approximate solution $\phi_{n}(x, \eta)$ depends on the unknown constant $\eta$. This constant $\eta$ can be obtained approximately by imposing the boundary condition at $x=1$ on $\phi_{n}(x, \eta)$, which leads to a sequence of transcendental equations $a \phi_{n}(1, \eta)+b \phi_{n}^{\prime}(1, \eta)=c n=$ $1,2,3, \ldots$ For example, consider

$$
y^{\prime \prime}(x)=-e^{y(x)}, \quad y(0)=y(1)=0 .
$$

According to the $\operatorname{ADM}(8)$, with $y_{0}(x, \eta)=\eta x$, we obtain the components as

$$
\begin{aligned}
y_{0}(x, \eta) & =\eta x, y_{1}(x, \eta) \\
& =\left(\frac{1}{\eta^{2}}-\frac{e^{\eta x}}{\eta^{2}}+\frac{x}{\eta}\right), y_{2}(x, \eta) \\
& =\left(-\frac{5}{4 \eta^{4}}+\frac{e^{\eta x}}{\eta^{4}}+\frac{e^{2 \eta x}}{4 \eta^{4}}-\frac{x}{2 \eta^{3}}-\frac{e^{\eta x} x}{\eta^{3}}\right),
\end{aligned}
$$

Consequently, the $n$-term approximate series solution can be obtained as

$$
\phi_{n}(x, \eta)=\sum_{j=0}^{n} y_{j}(x, \eta)
$$

By imposing the boundary condition at $x=1$ on $\phi_{n}(x, \eta)$, we obtain a sequence of transcendental equations $\phi_{n}(1, \eta)=0$, $n=1,2, \ldots$ as follows

$$
\begin{aligned}
\phi_{1}(1, \eta) \equiv \eta & +\left(\frac{1}{\eta^{2}}-\frac{e^{\eta}}{\eta^{2}}+\frac{1}{\eta}\right)=0, \\
\phi_{2}(1, \eta) \equiv \eta & +\left(\frac{1}{\eta^{2}}-\frac{e^{\eta}}{\eta^{2}}+\frac{1}{\eta}\right) \\
& +\left(-\frac{5}{4 \eta^{4}}+\frac{e^{\eta}}{\eta^{4}}+\frac{e^{2 \eta}}{4 \eta^{4}}-\frac{1}{2 \eta^{3}}-\frac{e^{\eta}}{\eta^{3}}\right)=0,
\end{aligned}
$$

However, solving such transcendental equations for $\eta$ requires additional computational work, and $\eta$ may not be uniquely determined. This may be the major disadvantage of the ADM for solving two-point boundary value problems. 


\section{The Proposed Recursive Scheme}

In this section, we propose a new efficient recursive scheme based on the ADM for solving nonlinear SBVPs (1). To overcome the singular behavior at $x=0$, we rewrite SBVPs (1) in operator form as

$$
\mathscr{L} y(x)=x^{\alpha} f(x, y(x)), \quad 0<x<1
$$

subject to the boundary conditions

$$
y^{\prime}(0)=0, \quad a y(1)+b y^{\prime}(1)=c,
$$

where $\mathscr{L}=d / d x\left(x^{\alpha} d / d x\right)$ is a second-order linear singular differential operator. Twofold integral operator $\mathscr{L}^{-1}[\cdot]$ regarded as the inverse operator of $\mathscr{L}$ is proposed as

$$
\mathscr{L}^{-1}[\cdot]=\int_{x}^{1} s^{-\alpha}\left[\int_{0}^{s}[\cdot] d x\right] d s .
$$

In order to establish the new efficient recursive scheme, we operate $\mathscr{L}^{-1}[\cdot]$ on left hand side of (14) and impose the boundary condition $y^{\prime}(0)=0$; we obtain

$$
\begin{aligned}
& \mathscr{L}^{-1}\left[\left(x^{\alpha} y^{\prime}(x)\right)^{\prime}\right] \\
& =\int_{x}^{1} s^{-\alpha}\left[\int_{0}^{s}\left(x^{\alpha} y^{\prime}(x)\right)^{\prime} d x\right] d s, \\
& =\int_{x}^{1} s^{-\alpha}\left[\int_{0}^{s}\left(x^{\alpha} y^{\prime \prime}(x)+\alpha x^{\alpha-1} y^{\prime}(x)\right) d x\right] d s, \\
& =\int_{x}^{1} s^{-\alpha}\left[s^{\alpha} y^{\prime}(s)-\int_{0}^{s} \alpha x^{\alpha-1} y^{\prime}(x) d x\right. \\
& \left.\quad+\int_{0}^{s} \alpha x^{\alpha-1} y^{\prime}(x) d x\right] d s, \\
& =\int_{x}^{1} y^{\prime}(s) d s=y(1)-y(x) .
\end{aligned}
$$

Thus we have

$$
\mathscr{L}^{-1}\left[\left(x^{\alpha} y^{\prime}(x)\right)^{\prime}\right]=c_{0}-y(x),
$$

where $c_{0}=y(1) \neq 0$ is unknown constant to be determined.

We now operate $\mathscr{L}^{-1}[\cdot]$ on both sides of (14) and use (18); we get

$$
y(x)=c_{0}-\int_{x}^{1} s^{-\alpha}\left[\int_{0}^{s} x^{\alpha} f(x, y(x)) d x\right] d s .
$$

To eliminate unknown constant $c_{0}$, we impose the boundary condition $a y(1)+b y^{\prime}(1)=c$ on (19) which leads to

$$
c_{0}=\frac{c}{a}-\frac{b}{a} \int_{0}^{1} x^{\alpha} f(x, y(x)) d x .
$$

Substituting the value of $c_{0}$ into (19), we obtain

$$
\begin{aligned}
y(x)= & \frac{c}{a}-\frac{b}{a} \int_{0}^{1} x^{\alpha} f(x, y(x)) d x \\
& -\int_{x}^{1} s^{-\alpha}\left[\int_{0}^{s} x^{\alpha} f(x, y(x)) d x\right] d s .
\end{aligned}
$$

Note that (21) does not involve any unknown constants to be determined.

We now decompose the solution $y(x)$ by the series as

$$
y(x)=\sum_{j=0}^{\infty} y_{j}(x), \quad f(x, y(x))=\sum_{j=0}^{\infty} A_{j},
$$

where $A_{n}$ are the Adomian's polynomials [13]. In 2010, Duan $[25,26]$ reported several new efficient algorithms for rapid computer generation of the Adomian polynomials. Recently, El-Kalla [27] suggested another programmable formula for Adomian polynomials:

$$
A_{n}=f\left(x, \psi_{n}\right)-\sum_{j=0}^{n-1} A_{j} \quad \text { or } f\left(x, \psi_{n}\right)=\sum_{j=0}^{n} A_{j},
$$

where $\psi_{n}=\sum_{j=0}^{n} y_{j}$ is partial sum of the series solution $\sum_{j=0}^{\infty} y_{j}$

Substituting the series (22) into (21), we obtain

$$
\begin{aligned}
\sum_{j=0}^{\infty} y_{j}(x)= & \frac{c}{a}-\frac{b}{a} \int_{0}^{1} x^{\alpha}\left[\sum_{j=0}^{\infty} A_{j}\right] d x \\
& -\int_{x}^{1} s^{-\alpha}\left[\int_{0}^{s} x^{\alpha} \sum_{j=0}^{\infty} A_{j} d x\right] d s .
\end{aligned}
$$

Comparing both sides of (24), the proposed scheme is given by the following recursive scheme:

$$
\begin{gathered}
y_{0}(x)=\frac{c}{a}, \\
y_{j}(x)=-\frac{b}{a} \int_{0}^{1} x^{\alpha} A_{j-1} d x-\int_{x}^{1} s^{-\alpha}\left[\int_{0}^{s} x^{\alpha} A_{j-1} d x\right] d s, \\
j \geq 1 .
\end{gathered}
$$

The recursive scheme (25) provides complete determination of solution components $y_{j}(x)$ of the solution $y(x)$. Hence, the truncated $n$-term series solution can be obtained as

$$
\psi_{n}(x)=\sum_{j=0}^{n} y_{j}(x)
$$

Unlike ADM or MADM, the proposed recursive scheme (25) does not require any computation of unknown constants.

\section{Convergence Analysis}

In this section, we will give the sufficient condition that guarantees existence of a unique solution of (28) in Theorem 1. Then we discuss the convergence analysis in Theorem 2 and the error analysis in Theorem 3 of the proposed scheme (25) for SBVPs (1). Note that many researcher [28-31] have also established the convergence of the ADM for solving 
differential as well as integral equations. Let $\mathbb{X}=C[0,1]$ be a Banach space with the norm

$$
\|y\|=\max _{x \in[0,1]}|y(x)|, \quad y \in \mathbb{X}
$$

Note that (21) can be written in operator form as

$$
y=\mathcal{N}(y)
$$

where $\mathcal{N}(y)$ is given by

$$
\begin{aligned}
\mathcal{N}(y)= & \frac{c}{a}-\frac{b}{a} \int_{0}^{1} x^{\alpha} f(x, y) d x \\
& -\int_{x}^{1} s^{-\alpha}\left[\int_{0}^{s} x^{\alpha} f(x, y) d x\right] d s .
\end{aligned}
$$

Theorem 1. Let $\mathbb{X}$ be a Banach space with the norm given by (27). Assuming that the nonlinear function $f(x, y)$ satisfies the Lipschitz condition; that is, $\left|f(x, y)-f\left(x, y^{*}\right)\right| \leq L\left|y-y^{*}\right|$. Further, let $\delta$ be a constant defined as

$$
\delta:=\frac{L(a+2 b)}{2 a(1+\alpha)}
$$

If $\delta<1$, then (28) has a unique solution in $\mathbb{X}$.

Proof. For any $y, y^{*} \in \mathbb{X}$, we have

$$
\begin{aligned}
& \left\|\mathcal{N}(y)-\mathcal{N}\left(y^{*}\right)\right\| \\
& =\max _{x \in[0,1]} \mid \frac{b}{a} \int_{0}^{1} x^{\alpha}\left[f(x, y)-f\left(x, y^{*}\right)\right] d x \\
& \quad+\int_{x}^{1} s^{-\alpha}\left[\int_{0}^{s} x^{\alpha}\left[f(x, y)-f\left(x, y^{*}\right)\right] d x\right] d s \mid, \\
& \leq \max _{x \in[0,1]}\left|f(x, y)-f\left(x, y^{*}\right)\right| \\
& \quad \times \max _{x \in[0,1]}\left|\frac{b}{a} \int_{0}^{1} x^{\alpha} d x+\int_{x}^{1} s^{-\alpha}\left[\int_{0}^{s} x^{\alpha} d x\right] d s\right| .
\end{aligned}
$$

Using Lipschitz continuity of $f$, we obtain

$$
\left\|\mathcal{N}(y)-\mathcal{N}\left(y^{*}\right)\right\| \leq \frac{L(a+2 b)}{2 a(1+\alpha)} \max _{x \in[0,1]}\left|y-y^{*}\right|=\delta\left\|y-y^{*}\right\|
$$

Hence, we have

$$
\left\|\mathcal{N}(y)-\mathcal{N}\left(y^{*}\right)\right\| \leq \delta\left\|y-y^{*}\right\|
$$

If $\delta<1$, then $\mathcal{N}: \mathbb{X} \rightarrow \mathbb{X}$ is contraction mapping and hence by the Banach contraction mapping theorem, (28) has a unique solution in $\mathbb{X}$.
We now prove the convergence of the proposed scheme (25). Let $\left\{\psi_{n}=\sum_{j=0}^{n} y_{j}\right\}$ be a sequence of partial sums of the series solution $\sum_{j=0}^{\infty} y_{j}$. Using (25) and (26), we have

$$
\begin{aligned}
\psi_{n}= & \sum_{j=0}^{n} y_{j}=y_{0}+\sum_{j=1}^{n} y_{j}=\frac{c}{a} \\
& -\sum_{j=1}^{n}\left[\frac{b}{a} \int_{0}^{1} x^{\alpha} A_{j-1} d x+\int_{x}^{1} s^{-\alpha}\left[\int_{0}^{s} x^{\alpha} A_{j-1} d x\right] d s\right] \\
= & \frac{c}{a}-\frac{b}{a} \int_{0}^{1} x^{\alpha} \sum_{j=1}^{n} A_{j-1} d x \\
& -\int_{x}^{1} s^{-\alpha}\left[\int_{0}^{s} x^{\alpha} \sum_{j=1}^{n} A_{j-1} d x\right] d s .
\end{aligned}
$$

Using (23) in (34), it follows that

$$
\begin{aligned}
\psi_{n}= & \frac{c}{a}-\frac{b}{a} \int_{0}^{1} x^{\alpha} f\left(x, \psi_{n-1}\right) d x \\
& -\int_{x}^{1} s^{-\alpha}\left[\int_{0}^{s} x^{\alpha} f\left(x, \psi_{n-1}\right) d x\right] d s,
\end{aligned}
$$

which is equivalent to the following operator equation:

$$
\psi_{n}=\mathcal{N}\left(\psi_{n-1}\right), \quad n=1,2, \ldots
$$

Note that the formulation (36) is used to prove Theorems 2 and 3 .

Theorem 2. Let $\mathcal{N}(y)$ be the nonlinear operator defined by (28) as contractive; that is,

$$
\begin{array}{r}
\left\|\mathcal{N}(y)-\mathcal{N}\left(y^{*}\right)\right\| \leq \delta\left\|y-y^{*}\right\|, \quad \forall y, y^{*} \in \mathbb{X} \\
\text { with } \delta<1,\left\|y_{1}\right\|<\infty .
\end{array}
$$

Then the sequence $\left\{\psi_{n}\right\}$ of partial sums defined by (26) converges to the exact solution $y$.

Proof. Using the relation (36) and the estimate (33), we have

$$
\begin{aligned}
& \left\|\psi_{m+1}-\psi_{m}\right\| \\
& \quad=\left\|\mathcal{N}\left(\psi_{m}\right)-\mathcal{N}\left(\psi_{m-1}\right)\right\| \leq \delta\left\|\psi_{m}-\psi_{m-1}\right\| .
\end{aligned}
$$

Thus we have

$$
\begin{aligned}
\left\|\psi_{m+1}-\psi_{m}\right\| & \leq \delta\left\|\psi_{m}-\psi_{m-1}\right\| \leq \delta^{2}\left\|\psi_{m-1}-\psi_{m-2}\right\| \\
& \leq \cdots \leq \delta^{m}\left\|\psi_{1}-\psi_{0}\right\| .
\end{aligned}
$$


We now show that the sequence $\left\{\psi_{n}\right\}$ is convergent. Now for all $n, m \in \mathbb{N}$, with $n \geq m$, we consider

$$
\begin{aligned}
& \left\|\psi_{n}-\psi_{m}\right\| \\
& \quad=\left\|\left(\psi_{n}-\psi_{n-1}\right)+\left(\psi_{n-1}-\psi_{n-2}\right)+\cdots+\left(\psi_{m+1}-\psi_{m}\right)\right\| \\
& \leq\left\|\psi_{n}-\psi_{n-1}\right\|+\left\|\psi_{n-1}-\psi_{n-2}\right\|+\cdots+\left\|\psi_{m+1}-\psi_{m}\right\| \\
& \quad \leq\left[\delta^{n-1}+\delta^{n-2}+\cdots+\delta^{m}\right]\left\|\psi_{1}-\psi_{0}\right\| \\
& =\delta^{m}\left[1+\delta+\delta^{2}+\cdots+\delta^{n-m-1}\right]\left\|\psi_{1}-\psi_{0}\right\| \\
& \quad=\delta^{m}\left(\frac{1-\delta^{n-m}}{1-\delta}\right)\left\|y_{1}\right\| .
\end{aligned}
$$

Since $0<\delta<1,\left(1-\delta^{n-m}\right)<1$, and $\left\|y_{1}\right\|<\infty$, it follows

$$
\left\|\psi_{n}-\psi_{m}\right\| \leq \frac{\delta^{m}}{1-\delta}\left\|y_{1}\right\| .
$$

Taking limit as $m \rightarrow \infty$, we obtain

$$
\left\|\psi_{n}-\psi_{m}\right\| \longrightarrow 0 \text {. }
$$

Hence $\left\{\psi_{n}\right\}$ is cauchy sequence in $\mathbb{X}$. Hence there exits $\psi$ in $\mathbb{X}$ such that $\lim _{n \rightarrow \infty} \psi_{n}=\psi$. Note that $\psi$ is the exact solution of (28) as

$$
y=\sum_{j=0}^{\infty} y_{j}=\lim _{n \rightarrow \infty} \psi_{n}=\psi .
$$

This completes the proof.

Theorem 3. Let $y(x)$ be the exact solution of the operator equation (28). Let $\psi_{m}(x)$ be the sequence of partial sums of series solution defined by (26). Then there holds

$$
\max _{x \in[0,1]}\left|y(x)-\sum_{j=0}^{m} y_{j}(x)\right| \leq \frac{M \delta^{m+1}}{L(1-\delta)},
$$

where $M=\max _{x \in[0,1]}\left|f\left(x, y_{0}\right)\right|$.

Proof. For any $n \geq m$ and using the estimate (41), we have

$$
\left\|\psi_{n}-\psi_{m}\right\| \leq \frac{\delta^{m}}{1-\delta}\left\|y_{1}\right\| .
$$

Since $\lim _{n \rightarrow \infty} \psi_{n}=y$, fixing $m$ and letting $n \rightarrow \infty$ in above estimate, we obtain

$$
\left\|y-\psi_{m}\right\| \leq \frac{\delta^{m}}{1-\delta} \max _{x \in[0,1]}\left|y_{1}(x)\right|
$$

Since, we have

$$
\begin{array}{r}
y_{1}(x)=-\frac{b}{a} \int_{0}^{1} x^{\alpha} A_{0} d x-\int_{x}^{1} s^{-\alpha}\left[\int_{0}^{s} x^{\alpha} A_{0} d x\right] d s \\
A_{0}=f\left(x, y_{0}\right),
\end{array}
$$

so

$$
\begin{aligned}
& \max _{x \in[0,1]}\left|y_{1}(x)\right| \\
& \quad=\max _{x \in[0,1]}\left|\frac{b}{a} \int_{0}^{1} x^{\alpha} A_{0} d x+\int_{x}^{1} s^{-\alpha}\left[\int_{0}^{s} x^{\alpha} A_{0} d x\right] d s\right| \\
& \quad \leq \frac{(a+2 b)}{2 a(1+\alpha)} \max _{x \in[0,1]}\left|f\left(x, u_{0}\right)\right| .
\end{aligned}
$$

Combining the estimates (46) and (48) and using $\delta / L=(a+$ $2 b) /(2 a(1+\alpha))$, we obtain

$$
\max _{x \in[0,1]}\left|y(x)-\sum_{j=0}^{m} y_{j}(x)\right| \leq \frac{M \delta^{m+1}}{L(1-\delta)} .
$$

This completes the proof.

\section{Numerical Examples}

In this section, we demonstrate the accuracy and reliability of the proposed scheme (25) by implementing it to four SBVPs, arising various physical models. All the numerical results obtained by the proposed (25) are compared with the results obtained by various numerical methods.

Example 1. Consider the following nonlinear SBVPs:

$$
\begin{gathered}
\left(x^{\alpha} y^{\prime}(x)\right)^{\prime}=x^{\alpha}\left(\frac{N_{1} y(x)}{y(x)+K_{1}}\right), \quad 0<x<1, \\
y^{\prime}(0)=0, \quad 5 y(1)+y^{\prime}(1)=5,
\end{gathered}
$$

where $N_{1}$ and $K_{1}$ are positive constants involving the reaction rate and Michaelis constants. Thus we take $N_{1}=0.76129$ and $K_{1}=0.03119$ as used in $[1,3,5]$.

According to the proposed method (25) with $a=5, b=$ 1 , and $c=5$. Consequently, we have the following recursive scheme:

$$
y_{0}(x)=1 \text {, }
$$

$$
\begin{aligned}
& y_{j}(x) \\
& =-\frac{1}{5} \int_{0}^{1} x^{\alpha} A_{j-1} d x-\int_{x}^{1} s^{-\alpha}\left[\int_{0}^{s} x^{\alpha} A_{j-1} d x\right] d s, \quad j \geq 1 .
\end{aligned}
$$

Using the formula (6), Adomian's polynomials for $f(y(x))=$ $0.76129 y(x) /(y(x)+0.03119)$ with $y_{0}=1$ are obtained as

$$
\begin{aligned}
A_{0}= & 0.738264, \\
A_{1}= & 0.02233 y_{1}(x), \\
A_{2}= & -0.0216546 y_{1}^{2}(x)+0.02233 y_{2}(x), \\
A_{3}= & 0.0209996 y_{1}^{3}(x)-0.0433091 y_{1}(x) y_{2}(x) \\
& +0.02233 y_{3}(x)
\end{aligned}
$$


Case ( $\alpha=1)$. Using (51) and (52), the components $y_{j}(x)$ of the solution $y(x)$ are obtained as

$$
\begin{aligned}
y_{0}(x) & =1 \\
y_{1}(x) & =-0.25839224+0.18456588 x^{2} \\
y_{2}(x) & =0.00155580-0.00144247 x^{2}+0.00025758 x^{4}, \\
y_{3}(x) & =0.00030986-0.0003527 x^{2}+0.0001270 x^{4} \\
& -0.0000203 x^{6}
\end{aligned}
$$

The 6-term approximate series solution $\psi_{6}(x)=\sum_{j=0}^{6} y_{j}(x)$ is given by

$$
\begin{aligned}
\psi_{6}(x)= & 0.743551+0.182662 x^{2}+0.0004499 x^{4} \\
& -0.0000453 x^{6}+5.181586 \times 10^{-6} x^{8} \\
& -5.061711 \times 10^{-7} x^{10}+2.801167 \times 10^{-8} x^{12} .
\end{aligned}
$$

Case $(\alpha=2)$. In a similar manner, we obtain the components $y_{j}(x)$ of solution $y(x)$ as

$$
\begin{aligned}
y_{0}(x) & =1 \\
y_{1}(x) & =-0.17226149+0.12304392 x^{2} \\
y_{2}(x) & =0.0006502-0.0006410 x^{2}+0.0001373 x^{4} \\
y_{3}(x) & =0.0000822-0.0001046 x^{2}+0.000045 x^{4} \\
& -7.732815 \times 10^{-6} x^{6}
\end{aligned}
$$

Hence, we obtain the 6-term approximate series solution $\psi_{6}(x)=\sum_{j=0}^{6} y_{j}$ as

$$
\begin{aligned}
\psi_{6}(x)= & 0.828483+0.122278 x^{2}+0.000196 x^{4} \\
& -0.000013 x^{6}+1.013102 \times 10^{-6} x^{8} \\
& -7.367823 \times 10^{-8} x^{10}+3.366698 \times 10^{-9} x^{12} .
\end{aligned}
$$

Case $(\alpha=3)$. The components $y_{j}(x)$ of the solution $y(x)$ are obtained as

$$
\begin{aligned}
y_{0}(x) & =1 \\
y_{1}(x) & =-0.1291961+0.0922829 x^{2} \\
y_{2}(x) & =0.000350-0.000360 x^{2}+0.0000858 x^{4} \\
y_{3}(x)= & 0.0000321-0.0000442 x^{2}+0.000021 x^{4} \\
& -3.802000 \times 10^{-6} x^{6}
\end{aligned}
$$

TABLE 1: Numerical results for $\alpha=0,1$ of Example 1 .

\begin{tabular}{ccccc}
\hline$x$ & $\psi_{6}, \alpha=0$ & Wazwaz [5] & $\psi_{6}, \alpha=1$ & Wazwaz [5] \\
\hline 0.0 & 0.4950040961 & 0.4952605157 & 0.7435513167 & 0.7435531556 \\
0.2 & 0.5093399331 & 0.5095882966 & 0.7508585027 & 0.7508602912 \\
0.4 & 0.5523907867 & 0.5526180550 & 0.7727885297 & 0.7727901915 \\
0.6 & 0.6242765760 & 0.6244730118 & 0.8093658234 & 0.8093673219 \\
0.8 & 0.7251677449 & 0.7253051125 & 0.8606280633 & 0.8606293419 \\
1.0 & 0.8552538780 & 0.8552148933 & 0.9266223859 & 0.9266231833 \\
\hline
\end{tabular}

TABLE 2: Numerical results for $\alpha=2$ of Example 1.

\begin{tabular}{ccccc}
\hline$x$ & $\psi_{4}$ & $\psi_{6}$ & Waswaz [5] & $\begin{array}{c}\text { Kanth and } \\
\text { Bhattacharya } \\
{[1]}\end{array}$ \\
\hline 0.0 & 0.8284816685 & 0.8284832618 & 0.8284832761 & 0.8284832730 \\
0.2 & 0.8333732324 & 0.8333747079 & 0.8333747193 & 0.8333747169 \\
0.4 & 0.8480515962 & 0.8480527660 & 0.8480527701 & 0.8480527704 \\
0.6 & 0.8725275171 & 0.8725283082 & 0.8725282997 & 0.8725283084 \\
0.8 & 0.9068180902 & 0.9068185418 & 0.9068185095 & 0.9068185403 \\
1.0 & 0.9509455924 & 0.9509457957 & 0.9509457539 & 0.9509457946 \\
\hline
\end{tabular}

TABLE 3: Numerical results for $\alpha=3,4,5$ of Example 1 .

\begin{tabular}{lcccc}
\hline$x$ & $\psi_{6}, \alpha=3$ & Waswaz [5] & $\psi_{6}, \alpha=4$ & $\psi_{6}, \alpha=5$ \\
\hline 0.0 & 0.8711896978 & 0.8711897615 & 0.8968770467 & 0.9140255488 \\
0.2 & 0.8748647597 & 0.8748648229 & 0.8998199822 & 0.9164795274 \\
0.4 & 0.8858920668 & 0.8858921290 & 0.9086501684 & 0.9238424346 \\
0.6 & 0.9042778592 & 0.9042779179 & 0.9233716779 & 0.9361171460 \\
0.8 & 0.9300321361 & 0.9300321834 & 0.9439910856 & 0.9533083270 \\
1.0 & 0.9631681076 & 0.9631681318 & 0.9705171710 & 0.9754222526 \\
\hline
\end{tabular}

The series solution is given as follows:

$$
\begin{aligned}
\psi_{6}(x)= & 0.87119+0.0918721 x^{2}+0.0001116 x^{4} \\
& -5.600223 \times 10^{-6} x^{6}+3.279844 \times 10^{-7} x^{8} \\
& -1.8874803 \times 10^{-8} x^{10}+7.358761 \times 10^{-10} x^{12} .
\end{aligned}
$$

Comparison of the numerical results obtained by the proposed method (25), the VIM used in [5], and the CSM used in [1] are shown in Tables 1, 2, and 3. These tables show that the numerical results obtained by the proposed scheme (25) are comparable with those in $[1,5]$. In addition, we have also presented numerical results for $\alpha=0,4,5$ in Tables 1 and 3 .

Example 2. Consider the following nonlinear SBVPs [9]:

$$
\begin{gathered}
\left(x^{2} y^{\prime}(x)\right)^{\prime}=x^{2} y^{5}(x), \quad 0<x<1, \\
y^{\prime}(0)=0, \quad y(1)=\frac{\sqrt{3}}{2} .
\end{gathered}
$$

The analytical solution is $y(x)=\sqrt{3 /\left(3+x^{2}\right)}$. 
According to the proposed method (25) with $\alpha=2, a=1$, $b=0$, and $c=\sqrt{3} / 2$. Consequently, we have the following recursive scheme as

$$
y_{0}(x)=\frac{\sqrt{3}}{2}
$$

$$
\begin{aligned}
& y_{j}(x) \\
& =-\frac{0}{1} \int_{0}^{1} x^{2} A_{j-1} d x-\int_{x}^{1} s^{-2}\left[\int_{0}^{s} x^{2} A_{j-1} d x\right] d s, \quad j \geq 1 .
\end{aligned}
$$

Similar to the previous example, Adomian's polynomials for $f(y(x))=y^{5}(x)$ are given as

$$
\begin{aligned}
A_{0}= & y_{0}^{5}(x), \\
A_{1}= & 5 y_{0}^{4} y_{1}(x), \\
A_{2}= & 10 y_{0}^{3}(x) y_{1}^{2}(x)+5 y_{0}^{4}(x) y_{2}(x), \\
A_{3}= & 10 y_{0}^{2}(x) y_{1}^{3}(x)+20 y_{0}^{3}(x) y_{1}(x) y_{2}(x) \\
& +5 y_{0}^{4}(x) y_{3}(x)
\end{aligned}
$$

Using (60) and (61), we obtain the components $y_{j}(x)$ of the solution $y(x)$ as

$$
\begin{aligned}
y_{0}(x)= & 0.8660254, y_{1}(x) \\
= & 0.0811899-0.0811899 x^{2}, y_{2}(x) \\
= & 0.0266404-0.0380578 x^{2}+0.0114173 x^{4}, y_{3}(x) \\
= & 0.0117741-0.0196235 x^{2}+0.00963337 x^{4} \\
& -0.00178396 x^{6}
\end{aligned}
$$

Hence, we obtain approximate series solution as

$$
\begin{aligned}
\psi_{6}(x)= & 0.996812-0.159895 x^{2}+0.0354173 x^{4} \\
& -0.00743284 x^{6}+0.00126204 x^{8} \\
& -0.000146626 x^{10}+8.48888 \times 10^{-6} x^{12} .
\end{aligned}
$$

For quantitative comparison, we now define the maximum absolute error by

$$
E_{n}=\max _{0<x \leq 1}\left|\psi_{n}(x)-y(x)\right| .
$$

Here $\psi_{n}(x)$ is the $n$-term approximate series solution obtained by the proposed method (25) and $y(x)$ is the analytical solution. Table 4 shows a comparison between the
TABLE 4: Comparison of the maximum absolute error of Example 2.

\begin{tabular}{lccc}
\hline$n$ & $E_{n}$ & $n$ & Chawla et al. [9] \\
\hline 5 & $5.0992 \times 10^{-3}$ & 16 & $3.64 \times 10^{-4}$ \\
10 & $9.2468 \times 10^{-5}$ & 32 & $2.49 \times 10^{-5}$ \\
15 & $2.1193 \times 10^{-6}$ & 64 & $1.60 \times 10^{-6}$ \\
\hline
\end{tabular}

TABLE 5: Comparison of the maximum absolute error of Example 3.

\begin{tabular}{lccccc}
\hline$n$ & $E_{n}$ & $n$ & Çağlar et al. [2] & $n$ & Chawla et al. [9] \\
\hline 5 & $6.2129 \times 10^{-4}$ & 20 & $3.1606 \times 10^{-5}$ & 16 & $2.52 \times 10^{-3}$ \\
10 & $1.1318 \times 10^{-6}$ & 40 & $7.8742 \times 10^{-6}$ & 32 & $1.83 \times 10^{-4}$ \\
15 & $2.0760 \times 10^{-7}$ & 60 & $3.5011 \times 10^{-6}$ & 64 & $1.28 \times 10^{-5}$ \\
\hline
\end{tabular}

numerical results obtained by the proposed method (25) and the FDM used in [9]. The numerical results obtained by the proposed method (25) show good agreement with those in [9].

Example 3. Consider the following nonlinear SBVPs [3]:

$$
\begin{gathered}
\left(x y^{\prime}(x)\right)^{\prime}=-x e^{y(x)}, \quad 0<x<1, \\
y^{\prime}(0)=0, \quad y(1)=0 .
\end{gathered}
$$

The analytical solution is $y(x)=2 \ln \left((C+1) /\left(C x^{2}+1\right)\right)$, where $C=3-2 \sqrt{2}$.

According to the proposed method (25) with $\alpha=1, a=1$, $b=0$, and $c=0$, we have the following recursive scheme:

$$
\begin{gathered}
y_{0}(x)=0, \\
y_{j}(x) \\
=\frac{0}{1} \int_{0}^{1} x A_{j-1} d x+\int_{x}^{1} s^{-1}\left[\int_{0}^{s} x A_{j-1} d x\right] d s, \quad j \geq 1 .
\end{gathered}
$$

Proceeding as before, Adomian's polynomials for $f(y(x))=$ $e^{y(x)}$ with $y_{0}(x)=0$ are given as

$$
\begin{aligned}
A_{0} & =1, A_{1} \\
& =y_{1}(x), A_{2} \\
& =\frac{y_{1}^{2}(x)}{2}+y_{2}(x), A_{3} \\
& =\frac{y_{1}^{3}(x)}{6}+y_{1}(x) y_{2}(x)+y_{3}(x)
\end{aligned}
$$


TABLE 6: Numerical results of Example 4.

\begin{tabular}{lccccr}
\hline$x$ & $\psi_{6}$ & $\psi_{8}$ & $\psi_{10}$ & Pandey [32] & Duggan and Goodman [8] \\
\hline 0.0 & 0.270736276 & 0.270258951 & 0.270108917 & - & 0.270350067 \\
0.2 & 0.265615467 & 0.265154126 & 0.265009271 & 0.264932820 & 0.265254341 \\
0.4 & 0.250163329 & 0.249747091 & 0.249616772 & 0.249548183 & 0.249867127 \\
0.6 & 0.224103659 & 0.223753983 & 0.223644912 & 0.223587710 & 0.223885976 \\
0.8 & 0.186952558 & 0.186680714 & 0.186596204 & 0.186552018 & 0.186798950 \\
1.0 & 0.137982462 & 0.137789790 & 0.137729980 & 0.137698751 & 0.137872638 \\
\hline
\end{tabular}

Making use of (66) and (67), we can calculate the components $y_{j}(x)$ of solution $y(x)$ as

$$
\begin{aligned}
y_{0}(x)= & 0, y_{1}(x) \\
= & 0.25-0.25 x^{2}, y_{2}(x) \\
= & 0.046875-0.0625 x^{2}+0.015625 x^{4}, y_{3}(x) \\
= & 0.0130208-0.0195313 x^{2}+0.0078125 x^{4} \\
& -0.00130208 x^{6}
\end{aligned}
$$

Hence we obtain approximate series solution as

$$
\begin{aligned}
\psi_{6}(x)= & 0.316294-0.342438 x^{2}+0.0289497 x^{4} \\
& -0.00310771 x^{6}+0.000328064 x^{8} \\
& -0.000027465 x^{10}+1.2715657 \times 10^{-6} x^{12} .
\end{aligned}
$$

Table 5 shows a comparison of the numerical results obtained by the proposed method (25), the FDM used in [9], and the BSM used in [2]. Once again, the approximate series solutions using proposed method (25) are comparable with the results reported earlier in the literature $[2,9]$.

Example 4. Consider the following nonlinear SBVPs [8]:

$$
\begin{gathered}
\left(x^{2} y^{\prime}(x)\right)^{\prime}=-x^{2} e^{-y(x)}, \quad 0<x<1, \\
y^{\prime}(0)=0, \quad 2 y(1)+y^{\prime}(1)=0 .
\end{gathered}
$$

According to the proposed method (25) with $\alpha=2, a=2$, $b=1$, and $c=0$, we have the following scheme:

$$
y_{0}(x)=0
$$

$$
\begin{aligned}
& y_{j}(x) \\
& \quad=\frac{1}{2} \int_{0}^{1} x^{2} A_{j-1} d x+\int_{x}^{1} s^{-2}\left[\int_{0}^{s} x^{2} A_{j-1} d x\right] d s, \quad j \geq 1 .
\end{aligned}
$$

Adomian's polynomials, for $f(y(x))=e^{-y(x)}$ with $y_{0}(x)=0$ are given as

$$
\begin{aligned}
& A_{0}=1, \\
& A_{1}=-y_{1}(x) \\
& A_{2}=\frac{y_{1}^{2}(x)}{2}-y_{2}(x), \\
& A_{3}=-\frac{y_{1}^{3}(x)}{6}+y_{1}(x) y_{2}(x)-y_{3}(x)
\end{aligned}
$$

Making use of (71) and (72), we obtain the component $y_{n}(x)$ as follows:

$$
\begin{aligned}
y_{0}(x)= & 0, y_{1}(x) \\
= & 0.333333-0.166667 x^{2}, y_{2}(x) \\
= & -0.0861111+0.0555556 x^{2}-0.00833333 x^{4}, y_{3}(x) \\
= & 0.032672-0.0236111 x^{2}+0.00555556 x^{4} \\
& -0.000529101 x^{6} \\
& \vdots
\end{aligned}
$$

Hence, the 6-term approximate series solution is given by

$$
\begin{aligned}
\psi_{6}(x)= & 0.270736-0.127835 x^{2}-0.00461112 x^{4} \\
& -0.00030756 x^{6}+3.17554 \times 10^{-6} x^{8} \\
& -3.22937 \times 10^{-6} x^{10}+2.18422 \times 10^{-7} x^{12} \\
& -1.74996 \times 10^{-8} x^{14}
\end{aligned}
$$

Table 6 presents a comparison of the approximate solutions obtained by the proposed method (25), the FDM used in [32], and tangent chord technique used in [8]. We again obtain the numerical results using the proposed scheme (25) which are comparable with those in $[8,32]$. 


\section{Conclusions}

(i) In this work, the simplicity, efficiency, and reliability of the proposed method (25) have been tested by solving four nonlinear SBVPs that arise in physiology.

(ii) The accuracy of the numerical results indicates that the proposed method is well suited for the solution of SBVPs. It has also been shown that only 6 terms are sufficient to obtain comparable solution with those in $[2,9]$.

(iii) Unlike ADM or MDAM, the proposed method (25) does not require any computation of unknown constants. In fact, it provides a direct scheme to obtain approximations to the solution.

(iv) Unlike the finite difference and the cubic spline methods, the proposed method does not require any linearization and discretization of the variables.

(v) Uniqueness of the solution of (28) has been discussed in Theorem 1 . The convergence analysis and the error estimation of the proposed scheme (25) have also been established in Theorems 2 and 3 .

\section{Conflict of Interests}

The authors declare that there is no conflict of interests regarding the publication of this paper.

\section{Acknowledgments}

The authors would like to thank the anonymous referees for their useful comments and suggestions that led to improvement of the presentation and content of this paper. The authors thankfully acknowledge the financial assistance provided by the Council of Scientific and Industrial Research (CSIR), New Delhi, India.

\section{References}

[1] A. S. V. R. Kanth and V. Bhattacharya, "Cubic spline for a class of non-linear singular boundary value problems arising in physiology," Applied Mathematics and Computation, vol. 174, no. 1, pp. 768-774, 2006.

[2] H. Çağlar, N. Çaǧlar, and M. Özer, "B-spline solution of nonlinear singular boundary value problems arising in physiology," Chaos, Solitons and Fractals, vol. 39, no. 3, pp. 1232-1237, 2009.

[3] S. A. Khuri and A. Sayfy, "A novel approach for the solution of a class of singular boundary value problems arising in physiology," Mathematical and Computer Modelling, vol. 52, no. 3-4, pp. 626-636, 2010.

[4] M. Kumar and N. Singh, "Modified Adomian decomposition method and computer implementation for solving singular boundary value problems arising in various physical problems," Computers and Chemical Engineering, vol. 34, no. 11, pp. 17501760, 2010.

[5] A.-M. Wazwaz, "The variational iteration method for solving nonlinear singular boundary value problems arising in various physical models," Communications in Nonlinear Science and Numerical Simulation, vol. 16, no. 10, pp. 3881-3886, 2011.
[6] S. H. Lin, "Oxygen diffusion in a spherical cell with nonlinear oxygen uptake kinetics," Journal of Theoretical Biology, vol. 60, no. 2, pp. 449-457, 1976.

[7] B. F. Gray, "The distribution of heat sources in the human head-theoretical consideration," Journal of Theoretical Biology, vol. 82, no. 3, pp. 473-476, 1980.

[8] R. C. Duggan and A. M. Goodman, "Pointwise bounds for a nonlinear heat conduction model of the human head," Bulletin of Mathematical Biology, vol. 48, no. 2, pp. 229-236, 1986.

[9] M. M. Chawla, R. Subramanian, and H. L. Sathi, "A fourth order method for a singular two-point boundary value problem," BIT Numerical Mathematics, vol. 28, no. 1, pp. 88-97, 1988.

[10] M. Kumar, "A difference method for singular two-point boundary value problems," Applied Mathematics and Computation, vol. 146, no. 2-3, pp. 879-884, 2003.

[11] A. Yıldırım and T. Öziş, "Solutions of singular IVPs of LaneEmden type by the variational iteration method," Nonlinear Analysis, Theory, Methods and Applications, vol. 70, no. 6, pp. 2480-2484, 2009.

[12] R. Singh and J. Kumar, "Solving a class of singular two-point boundary value problems using new modified decomposition method," ISRN Computational Mathematics, vol. 2013, Article ID 262863, 11 pages, 2013.

[13] G. Adomian and R. Rach, "Inversion of nonlinear stochastic operators," Journal of Mathematical Analysis and Applications, vol. 91, no. 1, pp. 39-46, 1983.

[14] G. Adomian and R. Rach, "A new algorithm for matching boundary conditions in decomposition solutions," Applied Mathematics and Computation, vol. 57, no. 1, pp. 61-68, 1993.

[15] G. Adomian and R. Rach, "Modified decomposition solution of linear and nonlinear boundary-value problems," Nonlinear Analysis: Theory, Methods, and Applications, vol. 23, no. 5, pp. 615-619, 1994.

[16] G. Adomian, Solving FrontIer Problems of Physics: the Decomposition Method, Kluwer Academic Publishers, 1994.

[17] A.-M. Wazwaz, "Approximate solutions to boundary value problems of higher order by the modified decomposition method," Computers and Mathematics with Applications, vol. 40, no. 6, pp. 679-691, 2000.

[18] A. M. Wazwaz, "A reliable algorithm for obtaining positive solutions for nonlinear boundary value problems," Computers and Mathematics with Applications, vol. 41, no. 10-11, pp. 12371244, 2001.

[19] M. Benabidallah and Y. Cherruault, "Application of the Adomian method for solving a class of boundary problems," Kybernetes, vol. 33, no. 1, pp. 118-132, 2004.

[20] M. Inc and D. J. Evans, "The decomposition method for solving of a class of singular two-point boundary value problems," International Journal of Computer Mathematics, vol. 80, no. 7, pp. 869-882, 2003.

[21] B. Jang, "Two-point boundary value problems by the extended Adomian decomposition method," Journal of Computational and Applied Mathematics, vol. 219, no. 1, pp. 253-262, 2008.

[22] A. Ebaid, "A new analytical and numerical treatment for singular two-point boundary value problems via the Adomian decomposition method," Journal of Computational and Applied Mathematics, vol. 235, no. 8, pp. 1914-1924, 2011.

[23] M. Inc, M. Ergüt, and Y. Cherruault, "A different approach for solving singular two-point boundary value problems," Kybernetes, vol. 34, no. 7-8, pp. 934-940, 2005. 
[24] A.-M. Wazwaz, "A new method for solving singular initial value problems in the second-order ordinary differential equations," Applied Mathematics and Computation, vol. 128, no. 1, pp. 45-57, 2002.

[25] J.-S. Duan, "Recurrence triangle for Adomian polynomials," Applied Mathematics and Computation, vol. 216, no. 4, pp. 12351241, 2010.

[26] J.-S. Duan, "An efficient algorithm for the multivariable Adomian polynomials," Applied Mathematics and Computation, vol. 217, no. 6, pp. 2456-2467, 2010.

[27] I. L. El-Kalla, "Error estimates for series solutions to a class of nonlinear integral equations of mixed type," Journal of Applied Mathematics and Computing, vol. 38, no. 1-2, pp. 341-351, 2012.

[28] K. Abbaoui and Y. Cherruault, "Convergence of Adomian's method applied to differential equations," Computers and Mathematics with Applications, vol. 28, no. 5, pp. 103-109, 1994.

[29] Y. Cherruault, "Convergence of Adomian's method," Kybernetes, vol. 18, no. 2, pp. 31-38, 1989.

[30] Y. Cherruault and G. Adomian, "Decomposition methods: a new proof of convergence," Mathematical and Computer Modelling, vol. 18, no. 12, pp. 103-106, 1993.

[31] M. M. Hosseini and H. Nasabzadeh, "On the convergence of Adomian decomposition method," Applied Mathematics and Computation, vol. 182, no. 1, pp. 536-543, 2006.

[32] R. K. Pandey, "A finite difference method for a class of singular two point boundary value problems arising in physiology," International Journal of Computer Mathematics, vol. 65, no. 1-2, pp. 131-140, 1997. 


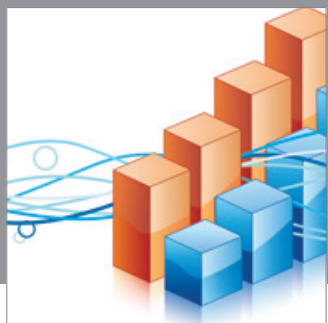

Advances in

Operations Research

mansans

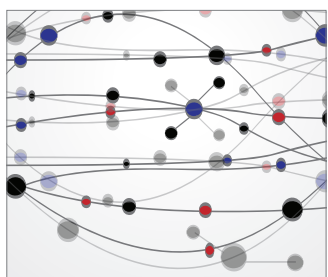

The Scientific World Journal
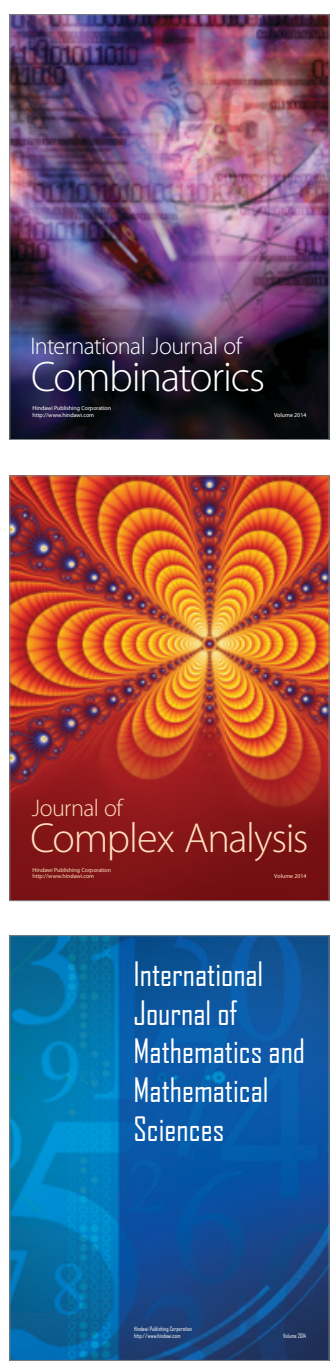
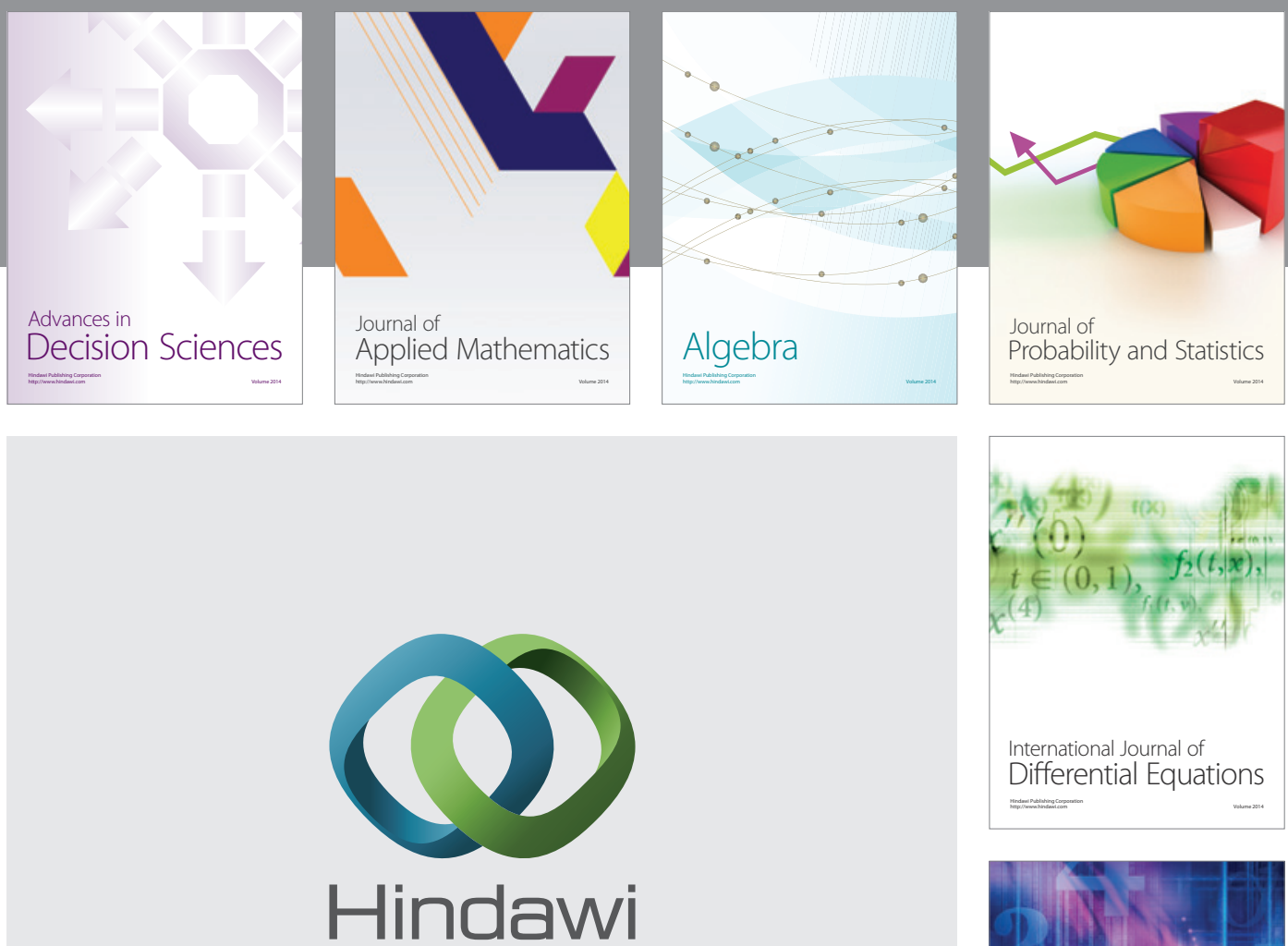

Submit your manuscripts at http://www.hindawi.com
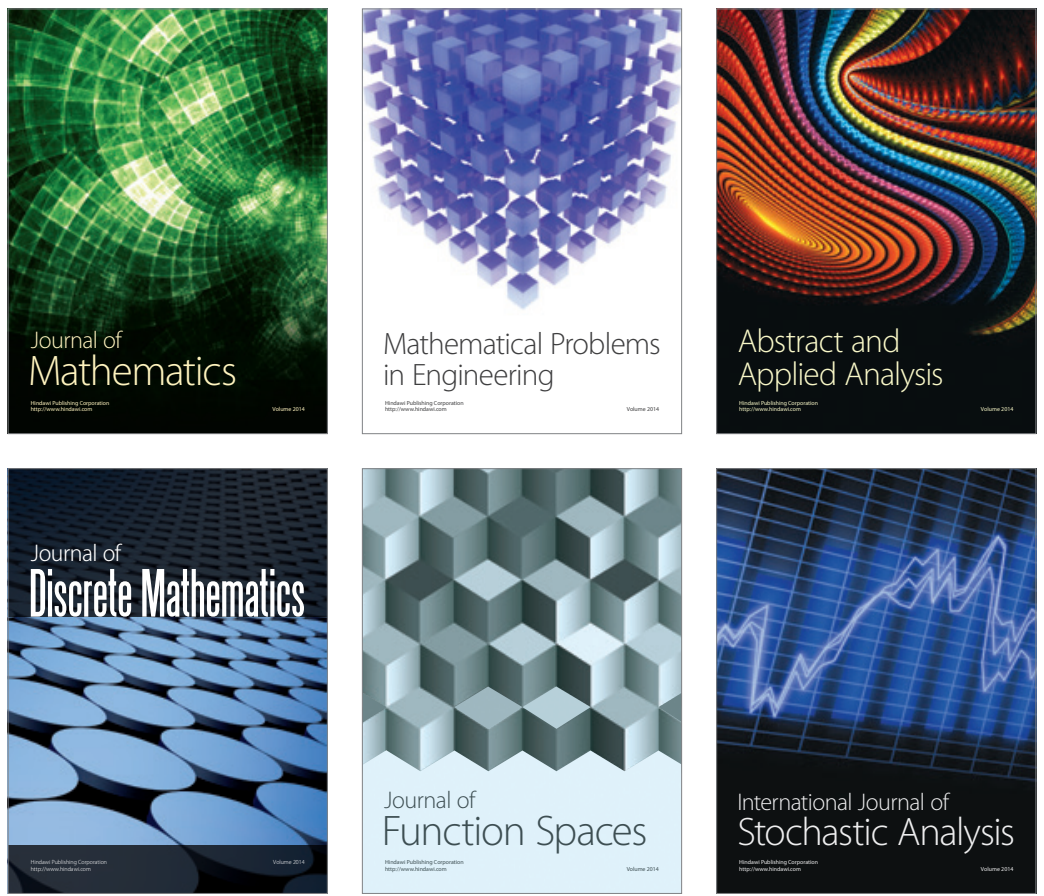

Journal of

Function Spaces

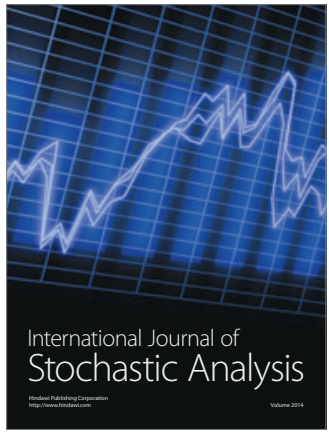

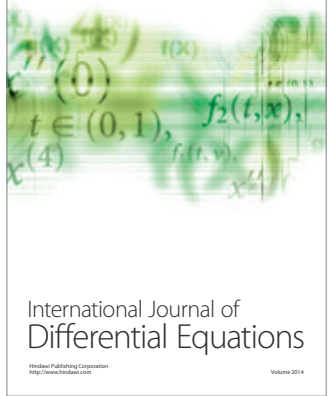
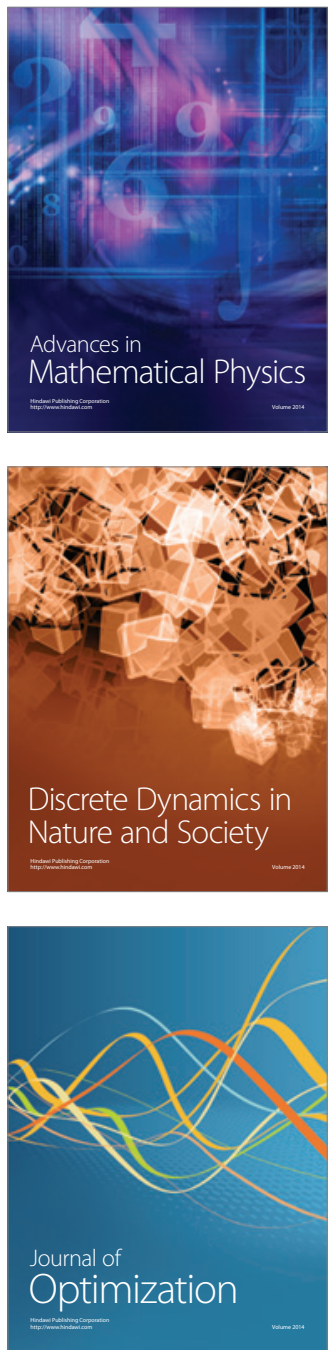